\title{
Automated radiogrammetry is a feasible method for measuring bone quality and bone maturation in severely disabled children
}

\author{
Sandra Mergler ${ }^{1,2}$ - Stella A. de Man $^{3}$ - Annemieke M. Boot ${ }^{4}$. \\ Karen G. C. B. Bindels-de Heus ${ }^{5}$ - Wim A. R. Huijbers ${ }^{6}$. Rick R. van Rijn ${ }^{7}$. \\ Corine Penning ${ }^{1} \cdot$ Heleen M. Evenhuis ${ }^{1}$
}

Received: 6 April 2015 /Revised: 26 December 2015 /Accepted: 13 January 2016 / Published online: 30 March 2016

(C) The Author(s) 2016. This article is published with open access at Springerlink.com

\begin{abstract}
Background Children with severe neurological impairment and intellectual disability are prone to low bone quality and fractures.

Objective We studied the feasibility of automated radiogrammetry in assessing bone quality in this specific group of children. We measured outcome of bone quality and, because these children tend to have altered skeletal maturation, we also studied bone age.

Materials and methods We used hand radiographs obtained in 95 children (mean age 11.4 years) presenting at outpatient paediatric clinics. We used BoneXpert software to determine bone quality, expressed as paediatric bone index and bone age.

Results Regarding feasibility, we successfully obtained a paediatric bone index in 60 children $(63.2 \%)$. The results on bone quality showed a mean paediatric bone
\end{abstract}

Sandra Mergler

s.mergler@erasmusmc.nl

1 Department of General Practice and Intellectual Disability Medicine, University Medical Centre, Erasmus MC,

Rotterdam, The Netherlands

2 Medical Department ASVZ, Care and Service Centre for People with Intellectual Disabilities, Sliedrecht, The Netherlands

3 Department of Paediatrics, Amphia Hospital,

Breda, The Netherlands

4 Department of Paediatric Endocrinology, University Medical Centre Groningen, index standard deviation score of -1.85 , significantly lower than that of healthy peers $(P<0.0001)$. Almost $50 \%$ of the children had severely diminished bone quality. In $64 \%$ of the children bone age diverged more than 1 year from chronological age. This mostly concerned delayed bone maturation.

Conclusion Automated radiogrammetry is feasible for evaluating bone quality in children who have disabilities but not severe contractures. Bone quality in these children is severely diminished. Because bone maturation frequently deviated from chronological age, we recommend comparison to bone-age-related reference values.

Keywords Automated radiogrammetry $\cdot$ Bone age $\cdot$ Bone maturation · Bone quality $\cdot$ Children · Disability .

Radiography
University of Groningen,

Groningen, The Netherlands

5 Department of General Paediatrics,

Sophia Children's Hospital,

University Medical Centre,

Erasmus MC,

Rotterdam, The Netherlands

6 Department of Paediatrics,

Beatrix Hospital,

Gorinchem, The Netherlands

7 Department of Radiology,

Emma Children's Hospital/Academic Medical Centre, Amsterdam, The Netherlands 


\section{Introduction}

Children with severe neurological impairment and intellectual disability are susceptible to developing low bone mineral density, which can lead to fractures originating from a limited or even unknown trauma [1-5].

In both adults and children, bone mineral density is generally measured with dual-energy X-ray absorptiometry [3, 4, 6]. Measurement in children requires specific software and adapted reference values [7, 8]. In the Netherlands, paediatric dual-energy X-ray absorptiometry is only available at tertiary care centres, which restricts the use of dual-energy X-ray absorptiometry in this specific group of children. In addition, disrupting factors can negatively influence the reliability of dual-energy X-ray absorptiometry results; these factors include contractures, scoliosis and movement during measurement, which are common in this group of children [9]. Therefore it is important in this group of children at risk for low bone mineral density to find a reliable diagnostic method of screening bone quality that is generally available at hospitals, easy to apply and less prone to errors caused by disrupting factors than dual-energy X-ray absorptiometry.

With automated radiogrammetry of plain hand radiographs, both bone quality and bone age can be measured with web-based software [10]. Bone quality measured with automated radiogrammetry is expressed as paediatric bone index. This paediatric bone index is determined by geometrical calculations, similar to the determination of digital X-ray radiogrammetry bone mineral density. The digital X-ray radiogrammetry bone mineral density has been shown to correlate well with peripheral dual-energy X-ray absorptiometry measurements in studies of the forearm, the femoral neck in adults, and the lumbar spine and total body in children [10-13]. Paediatric bone index reference values have been developed in a large group of healthy children $(n=2,398)$ [10]. These reference values are related to gender and bone age [10]. In children treated for acute lymphoblastic leukaemia and growth hormone deficiency, automated radiogrammetry has been shown to be easy to apply using a negligible effective radiation dose [13]. However, no data have been published on the use of this method in children with severe neurological impairment and intellectual disability.

Bone age can be determined automatically based on either the Greulich and Pyle or the Tanner and Whitehouse reference [14]. Age-related reference values are generally used in diagnostic methods (such as dual-energy X-ray absorptiometry and quantitative ultrasound [US]) used to assess bone quality in children [15].

In clinical practice, severely disabled children frequently have compromised growth velocity, whereas their skeletal maturation can be either delayed or accelerated [2, 16-18]. This evokes the question whether a difference exists between bone age and chronological age in children with severe neurological impairment and intellectual disability. Therefore the aims of this study were to determine the feasibility of automated radiogrammetry in children with severe neurological impairment and intellectual disability, and to obtain results on bone quality and bone age in this group of children. Additionally, we assessed differences between bone age and chronological age in this group of severely disabled children.

\section{Materials and methods}

\section{Study design}

This study was part of a cross-sectional multicentre study on bone quality in children with severe neurological impairment and intellectual disability. Four Dutch hospitals participated. Together these four hospitals cover a large part of the southwest of the Netherlands. The ethics committees of the Erasmus University Medical Center Rotterdam (MEC-2005182 ) and of each participating hospital approved this study.

\section{Study population}

We included children with severe neurological impairment and intellectual disability. These children were known to have a moderate or severe intellectual disability (intelligence quotient $<55$ ) and a gross motor functioning classification system level IV or V; all children visited the outpatient paediatric clinic of a participating hospital. The five-level Gross Motor Function Classification System is widely used for children with cerebral palsy and describes gross motor function on the basis of self-initiated movement [19]. Children in level IV may walk short distances with physical assistance of an adult at home but use wheeled mobility outdoors. Children in level $\mathrm{V}$ always use a wheelchair for mobility and have severe limitations in head and trunk control $[19,20]$.

Children were subdivided into five groups according to the aetiology of their disability. The first group consisted of children with a congenital cause, for example lissencephaly; the second group had perinatal complications, for example placental abruption; the third group had acquired disabilities like meningitis or trauma; the fourth group included children with a combination of congenital and acquired causes; the final group included children with an unknown cause of disability. Ninety-five children were included from June 2006 until January 2009.

\section{Automated radiogrammetry}

Automated radiogrammetry analysis was usually performed on a posterior anterior radiograph of the non-dominant hand. In cases where fewer contractures were present in the right hand, the right hand was used. To get optimal radiographic 
results, one of the authors (S.M., with 10 years of experience in Intellectual Disability Medicine (ID-medicine)) was present during all radiographic examinations. A maximum of two radiographs were taken. The preferred method was digital radiography in DICOM format, but traditional film radiographs could also be used once digitised using the Diagnostic Pro Advantage Scanner (VIDAR Systems Corp., Herndon, VA).

Automated radiogrammetry was performed with BoneXpert $\odot$ (software version 1.14; Visiana, Holte, Denmark). This method determines the bone ages of the middle three metacarpals and a minimum of eight other hand bones (Fig. 1).

\section{Feasibility}

Feasibility was specified in terms of successful determination of paediatric bone index. The authors considered the method feasible if the paediatric bone index standard deviation score could be obtained in at least $70 \%$ of the children.

\section{Bone quality}

Paediatric bone index was calculated using the three middle metacarpals by a formula containing the average values for transverse cortical area (A), bone width (W) and bone length (L): paediatric bone index $=\mathrm{A} /\left(\mathrm{W}^{1.33} \mathrm{~L}^{0.33}\right)$ [10]. Individual paediatric bone index outcomes were compared to reference values determined in healthy bone age- and gender- matched children and expressed in a standard deviation score [10].

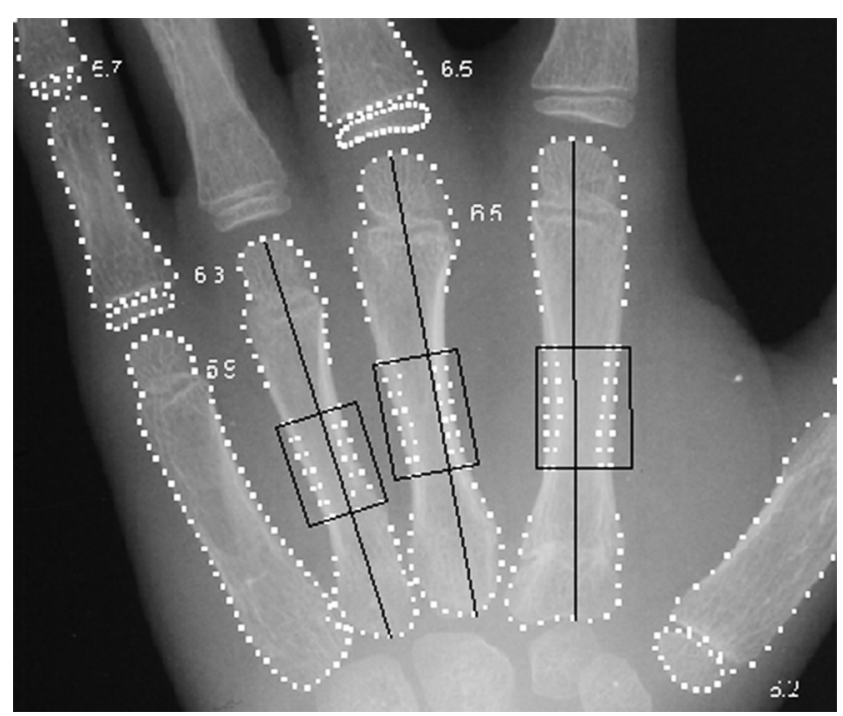

Fig. 1 Bone age. Hand radiograph of a 6-year-old girl shows the bone borders used for calculating bone age (dotted white lines around the individual bones, or ossification centres) and paediatric bone index (black boxes in metacarpals II through IV), as outlined by BoneXpert. The small numbers represent the given bone age for the individual bones used in calculating bone age

\section{Bone age}

BoneXpert can automatically determine either the Greulich and Pyle bone age or the Tanner and Whitehouse bone age. In this study we used the Greulich and Pyle reference, which has been found to be a robust method of automatic determination of bone age [14]. Valid and consistent bone ages of at least eight bones were required to assess bone age [21]. The difference between chronological age and bone age was calculated as automated bone age (years) minus chronological age (years). Based on clinical experience, a difference of 1 year or more in either direction was defined as relevant.

\section{Statistical analysis}

Statistical analysis was performed using Statistical Package for Social Sciences for Windows 15.0 (IBM, Armonk, NY). Results were expressed as mean \pm standard deviation. We used Student's $t$-tests and Pearson chi-square tests to calculate differences between groups (children with a measurable paediatric bone index standard deviation score versus children with no measurable paediatric bone index standard deviation score). A $P$-value of less than 0.05 was considered statistically significant.

\section{Results}

Patient characteristics are summarized in Table 1. We included 95 children with a mean age of 11.4 years (standard deviation [SD] 4.8 years); 53 (55.8\%) were male. Eighty percent of the children had a gross motor functioning classification level V. The most common causes of disability were congenital (40\%) and perinatal (31\%). Mean weight of the children was 32.3 kilograms (range $19.7-44.7 \mathrm{~kg}$ ), and $82.1 \%$ of the children had epilepsy.

\section{Feasibility}

Hand radiographs were obtained from all 95 children. The paediatric bone index standard deviation score could be calculated from 60 radiographs $(63.2 \%$; $95 \%$ confidence interval $53.5-72.9 \%)$. Bone age determination was not possible in 35 children. Unsuccessful measurements had various causes (Table 2). The most common cause was contractures of the hand, which resulted in crossed projection of the metacarpals on the radiograph (Fig. 2).

Assessment of bone quality was more frequently unsuccessful in children with more severe motor disabilities, scored as gross motor functioning classification system level $\mathrm{V}$ (chisquare test, $P=0.03$ ). Age, gender, aetiology of the disability, weight and epilepsy did not influence feasibility. 
Table 1 Characteristics of the study population $(n=95)$

\begin{tabular}{lll}
\hline Age $^{\mathrm{a}}$ in years & & $11.4(4.8)$ \\
Weight $^{\mathrm{a}}$ in kilograms & & $32.3(12.5)$ \\
Gender & Male/female & $53 / 42$ \\
Epilepsy & & 78 \\
$\begin{array}{l}\text { Gross motor functioning } \\
\text { classification system }\end{array}$ & Level IV/V & $19 / 76$ \\
Aetiology of disability & Congenital & 38 \\
& Perinatal & 29 \\
& Acquired & 7 \\
& Combination of congenital & 4 \\
& and acquired & 17 \\
& Unknown & \\
\hline
\end{tabular}

${ }^{\mathrm{a}}$ mean with standard deviation in parentheses

\section{Bone quality}

The mean paediatric bone index standard deviation score in these 60 children was -1.85 (SD 1.9), significantly lower than that of healthy peers with the same bone age $(P<0.0001)$. There was no difference in mean paediatric bone index standard deviation score between boys and girls $(P=0.35)$. Paediatric bone index standard deviation score was not associated with age $(P=0.84)$. In $29 / 60$ children $(48.3 \%)$ the paediatric bone index standard deviation score was less than -2.0 .

\section{Bone age}

To determine bone age successfully, images of at least eight hand and wrist bones were required. Bone age could not be measured in four children. Bone age and chronological age diverged more than 1 year in 36/56 (64\%; 95\% CI 51.7$76.8 \%$ ) children. Bone maturation was delayed in 26 children and accelerated in 10. Individual differences between both values varied from a bone age 3 years ahead of chronological age to 6 years behind (Fig. 3 ).

\section{Discussion}

This is a unique study using automated radiogrammetry in children with severe neurological impairment and intellectual disability. We assessed feasibility of using radiogrammetry to determine bone quality and bone age in this severely disabled group. We successfully determined bone index standard deviation score in 60 of $95(63.2 \%)$ children with severe neurological impairment and intellectual disability. This percentage is slightly lower than the minimum of $70 \%$ we required for the method to be considered feasible for this group of children. Bone quality determination was more difficult in children with a gross motor functioning classification system level V. These children have severe contractures, which was the most common cause for unsuccessful measurement (17/35, 49\%). This made correct identification of bone edges, necessary for automated assessment of the paediatric bone index, impossible. Although this reduces the usability of the automated radiogrammetry method in the most severely disabled paediatric group, it is important to realise that in children with severe contractures and deviant posture other diagnostic methods are also difficult to apply [9, 22].

The mean paediatric bone index standard deviation score in this group of children was -1.84 (SD: 1.93) and almost half of the children had a paediatric bone index standard deviation score lower than -2 , demonstrating that bone quality is clearly diminished in children with severe neurological impairment and intellectual disability in comparison to healthy children with similar bone age and gender [23]. Measurements failed more often in children with more severe motor disability. Motor dysfunction is an important risk factor of low bone density [23]. Therefore at least some of the children in whom the measurement failed could have been diagnosed with (very) low paediatric bone index. Accordingly, the actual frequency of low bone density in the overall group may be even higher.

The BoneXpert method uses automated bone age determination, preventing interobserver variation. Bone age and chronological age were found to diverge in a substantial number of children; the difference was more than 1 year in 36/56 children (64.3\%). Comparing the paediatric bone index outcome value to bone-age-related reference values appears to result in more accurate outcome measures, because skeletal growth and maturation, and bone mineral accrual appear to be closely related [24]. These important aspects of growth in children with severe neurological impairment and intellectual disability might
Table 2 Reasons for failure to obtain paediatric bone index standard deviation score $(n=35)$

\begin{tabular}{llc}
\hline Reason for failure & Number & Percentage \\
\hline Missing bone age & 2 & 5.7 \\
Contractures of the hand causing crossed projection of the metacarpals (Fig. 2) & 17 & 48.6 \\
$\begin{array}{l}\text { Excessive sharpening giving lack of contrast between bone tissue and surrounding } \\
\quad \text { soft tissue }\end{array}$ & 8 & 22.9 \\
$\begin{array}{l}\text { Anatomical deformities of the bones (not possible to determine exact margins of } \\
\quad \text { regions of interest) }\end{array}$ & 3 & 8.6 \\
Unclear & 5 & 14.3 \\
\hline
\end{tabular}




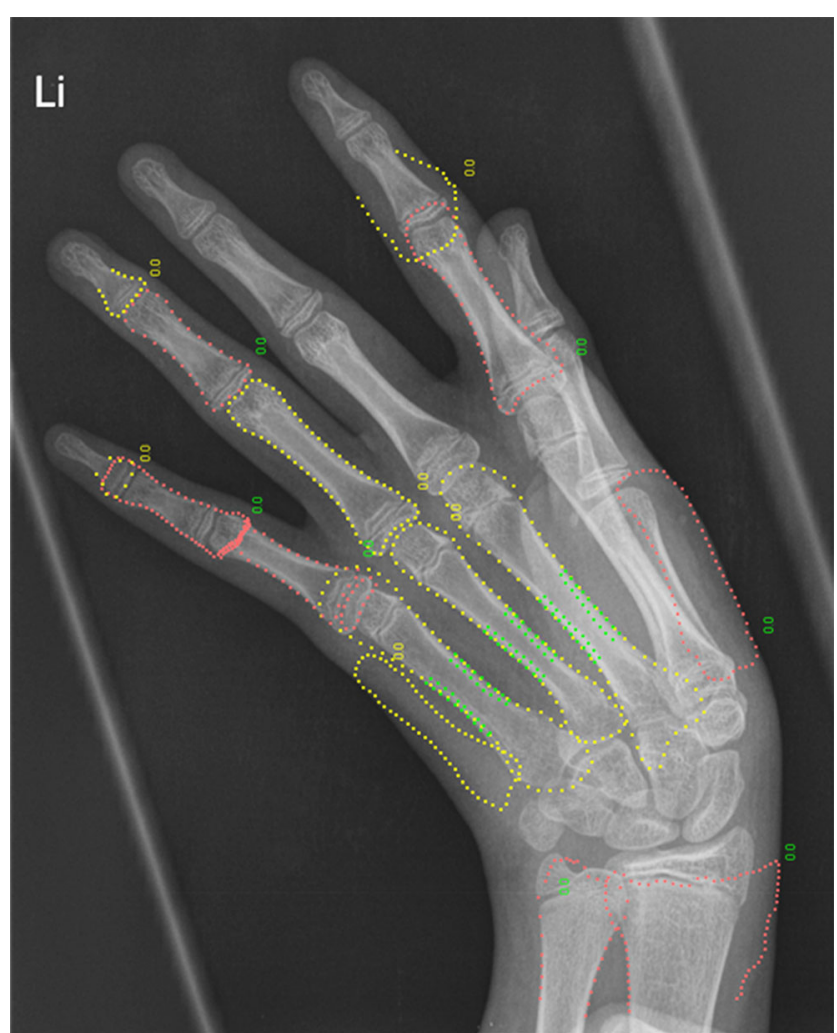

Fig. 2 Failed bone age determination using a hand radiograph in a 13year-old girl with projection of the metacarpals. Hand contractures causing overprojection of the metacarpals prevented BoneXpert software from obtaining the correct positioning of the dotted lines on the bone edges and correct positioning of the regions of interest in this case. $L i$ left

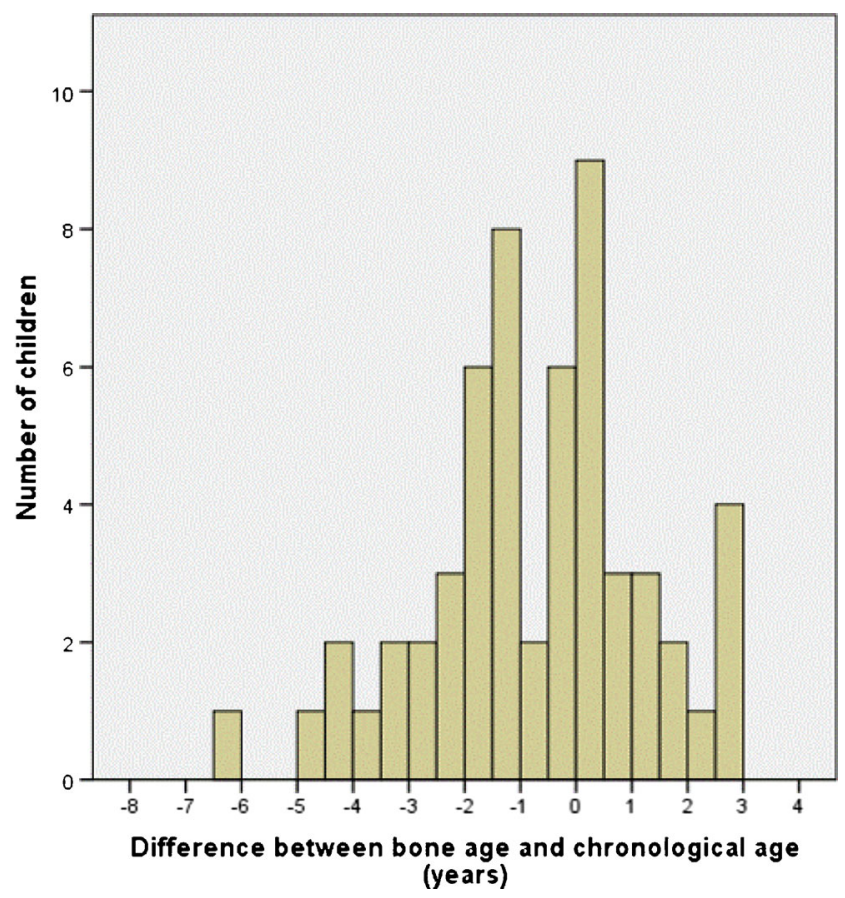

Fig. 3 Histogram shows differences between chronological age and bone age $(n=56)$. Chronological age is defined as age in years at time of measurement. Bone age is the automated bone age in years as determined by BoneXpert software based on hand radiographs similarly hinder other diagnostic methods of bone mineral density and bone quality [15].

Children with severe neurological impairment and intellectual disability compose a small but vulnerable group of patients requiring frequent medical care and interventions at hospitals and other care centres. Therefore study of these children is limited by the small number of patients in this group. However, fractures associated with compromised bone health are frequently present in this group. In addition to an increasing life expectancy in these children resulting from improved health care (for example by introduction of the gastrostomy catheters that improve nutritional state), an increasing fracture incidence can be expected. Therefore automated radiogrammetry might be helpful in the long-term evaluation of bone quality in this group.

\section{Conclusion}

Automated radiogrammetry is a feasible method to determine paediatric bone index in children with severe neurological impairment and intellectual disability but without severe contractures. Bone quality is clearly diminished in this group of children in comparison to healthy children with the same bone age and gender. Also, bone age and chronological age were found to diverge in a substantial number of the children. Bone age should be taken into account when other diagnostic methods (for example dual-energy X-ray absorptiometry and quantitative ultrasound) are used to determine bone quality in this group.

Acknowledgments We thank Mariëlle Bakker for assistance with the data collection, all participating children and parents for their cooperation, and the participating hospitals and hospital staff of the Erasmus University Medical Center Sophia Rotterdam, Albert Schweitzer Hospital Dordrecht, Beatrix Hospital Gorinchem and Amphia Hospital Breda for their hospitality and cooperation with our study. This study would not have been possible without the financial support we received from ASVZ and the Phelps-Stichting.

\section{Compliance with ethical standards}

Conflicts of interest None

Open Access This article is distributed under the terms of the Creative Commons Attribution 4.0 International License (http:// creativecommons.org/licenses/by/4.0/), which permits unrestricted use, distribution, and reproduction in any medium, provided you give appropriate credit to the original author(s) and the source, provide a link to the Creative Commons license, and indicate if changes were made.

\section{References}

1. Liptak GS, O'Donnell M, Conaway M et al (2001) Health status of children with moderate to severe cerebral palsy. Dev Med Child Neurol 43:364-370 
2. Stevenson RD, Conaway M, Chumlea WC et al (2006) Growth and health in children with moderate-to-severe cerebral palsy. Pediatrics 118:1010-1018

3. King W, Levin R, Schmidt R et al (2003) Prevalence of reduced bone mass in children and adults with spastic quadriplegia. Dev Med Child Neurol 45:12-16

4. Henderson RC, Lark RK, Gurka MJ et al (2002) Bone density and metabolism in children and adolescents with moderate to severe cerebral palsy. Pediatrics 110:e5

5. Brunner R, Doderlein L (1996) Pathological fractures in patients with cerebral palsy. J Pediatr Orthop B 5:232-238

6. Dreyfus D, Lauer E, Wilkinson J (2014) Characteristics associated with bone mineral density screening in adults with intellectual disabilities. J Am Board Fam Med 27:104-114

7. Binkovitz LA, Henwood MJ, Sparke P (2007) Pediatric dualenergy X-ray absorptiometry: technique, interpretation, and clinical applications. Semin Nucl Med 37:303-313

8. Van der Sluis IM, de Ridder MA, Boot AM et al (2002) Reference data for bone density and body composition measured with dual energy $\mathrm{x}$ ray absorptiometry in white children and young adults. Arch Dis Child 87:341-347

9. Mergler S, Rieken R, Tibboel D et al (2012) Lumbar spine and total-body dual-energy X-ray absorptiometry in children with severe neurological impairment and intellectual disability: a pilot study of artefacts and disrupting factors. Pediatr Radiol 42:574-583

10. Thodberg HH, van Rijn RR, Tanaka T et al (2010) A paediatric bone index derived by automated radiogrammetry. Osteoporos Int 21:1391-1400

11. Rosholm A, Hyldstrup L, Backsgaard L et al (2001) Estimation of bone mineral density by digital X-ray radiogrammetry: theoretical background and clinical testing. Osteoporos Int 12:961-969

12. Dhainaut A, Rohde GE, Syversen U et al (2010) The ability of hand digital X-ray radiogrammetry to identify middle-aged and elderly women with reduced bone density, as assessed by femoral neck dual-energy X-ray absorptiometry. J Clin Densitom 13:418-425

13. Van Rijn RR, Boot A, Wittenberg R et al (2006) Direct X-ray radiogrammetry versus dual-energy $\mathrm{X}$-ray absorptiometry: assessment of bone density in children treated for acute lymphoblastic leukaemia and growth hormone deficiency. Pediatr Radiol 36:227-232

14. Van Rijn RR, Lequin MH, Thodberg HH (2009) Automatic determination of Greulich and Pyle bone age in healthy Dutch children. Pediatr Radiol 39:591-597

15. Van Rijn RR, Van Kuijk C (2009) Of small bones and big mistakes; bone densitometry in children revisited. Eur J Radiol 71:432-439

16. Kuperminc MN, Gurka MJ, Houlihan CM et al (2009) Puberty, statural growth, and growth hormone release in children with cerebral palsy. J Pediatr Rehabil Med 2:131-141

17. Henderson RC, Gilbert SR, Clement ME et al (2005) Altered skeletal maturation in moderate to severe cerebral palsy. Dev Med Child Neurol 47:229-236

18. Gilbert SR, Gilbert AC, Henderson RC (2004) Skeletal maturation in children with quadriplegic cerebral palsy. J Pediatr Orthop 24: 292-297

19. Rosenbaum PL, Palisano RJ, Bartlett DJ et al (2008) Development of the gross motor function classification system for cerebral palsy. Dev Med Child Neurol 50:249-253

20. Hanna SE, Rosenbaum PL, Bartlett DJ et al (2009) Stability and decline in gross motor function among children and youth with cerebral palsy aged 2 to 21 years. Dev Med Child Neurol 51:295-302

21. Thodberg HH, Kreiborg S, Juul A et al (2009) The BoneXpert method for automated determination of skeletal maturity. IEEE Trans Med Imaging 28:52-66

22. Mergler S, Lobker B, Evenhuis HM et al (2010) Feasibility of quantitative ultrasound measurement of the heel bone in people with intellectual disabilities. Res Dev Disabil 31:1283-1290

23. Mergler S, Evenhuis HM, Boot AM et al (2009) Epidemiology of low bone mineral density and fractures in children with severe cerebral palsy: a systematic review. Dev Med Child Neurol 51: 773-778

24. Houlihan CM, Stevenson RD (2009) Bone density in cerebral palsy. Phys Med Rehabil Clin N Am 20:493-508 